This is the author's final, peer-reviewed manuscript as accepted for publication. The publisher-formatted version may be available through the publisher's web site or your institution's library.

\title{
Common dyadic coping is indirectly related to dietary and exercise adherence via patient and partner diabetes efficacy
}

Matthew D. Johnson, Jared R. Anderson, Ann Walker, Allison Wilcox, Virginia L. Lewis, and David C. Robbins

\section{How to cite this manuscript}

If you make reference to this version of the manuscript, use the following information:

Johnson, M. D., Anderson, J. R., Walker, A., Wilcox, A., Lewis, V. L., \& Robbins, D. C. (2013). Common dyadic coping is indirectly related to dietary and exercise adherence via patient and partner diabetes efficacy. Retrieved from http://krex.ksu.edu

\section{Published Version Information}

Citation: Johnson, M. D., Anderson, J. R., Walker, A., Wilcox, A., Lewis, V. L., \& Robbins, D. C. (2013). Common dyadic coping is indirectly related to dietary and exercise adherence via patient and partner diabetes efficacy. Journal of Family Psychology, 27(5), 722-730.

Copyright: (2013 American Psychological Association

Digital Object Identifier (DOI): doi:10.1037/a0034006

Publisher's Link: http://psycnet.apa.org/journals/fam/27/5/722/

This item was retrieved from the K-State Research Exchange (K-REx), the institutional repository of Kansas State University. K-REx is available at http://krex.ksu.edu 
Common Dyadic Coping Is Indirectly Related to Dietary and Exercise Adherence via Patient and Partner Diabetes Efficacy

\author{
Matthew D. Johnson \\ University of Alberta \\ Jared R. Anderson \\ Kansas State University
}

Ann Walker, Allison Wilcox, Virginia L. Lewis, and David C. Robbins

University of Kansas Medical Center

REDCap at University of Kansas Medical Center is supported by CTSA grant (Award \# UL1TR000001) from NCRR and NCATS awarded to the University of Kansas Medical Center for Frontiers: The Heartland Institute for Clinical and Translational Research

*Address correspondence to: Matthew D. Johnson, Ph.D., University of Alberta, 302 Human Ecology Building, Edmonton, AB Canada, T6G 0P9. Phone: (780) 492-5008. Fax: (780) 4924821. Email: matt.johnson@ualberta.ca 


\begin{abstract}
Using cross-sectional data from 117 married couples in which one member is diagnosed with type 2 diabetes, the current study sought to explore a possible indirect association between common dyadic coping and dietary and exercise adherence via the mechanism of patient and spouse reports of diabetes efficacy. Results from the structural equation model analysis indicated common dyadic coping was associated with higher levels of diabetes efficacy for both patients and spouses which, in turn, was then associated with better dietary and exercise adherence for the patient. This model proved a better fit to the data than three plausible alternative models. The bootstrap test of mediation revealed common dyadic coping was indirectly associated with dietary adherence via both patient and spouse diabetes efficacy, but spouse diabetes efficacy was the only mechanism linking common dyadic coping and exercise adherence. This study highlights the importance of exploring the indirect pathways through which general intimate relationship functioning might be associated with type 2 diabetes outcomes.

Keywords: dyadic coping, type 2 diabetes, couple relationship, dietary adherence, exercise adherence, chronic illness
\end{abstract}


Type 2 diabetes mellitus is a chronic health condition afflicting nearly 350 million people worldwide, a figure that has more than doubled over the last 30 years (Danaei et al., 2011). Health care expenditures are 2.3 times higher for those diagnosed with diabetes and total direct medical costs for treating diabetes in the United States were \$116 billion in 2007 (Centers for Disease Control and Prevention, 2011). In addition to pharmacological intervention, the successful management of type 2 diabetes requires adherence to a strict dietary and exercise regimen (Wing et al., 2001). Failure to maintain proper glycemic control can result in serious complications, including renal failure, neuropathy, vision problems, and cardiovascular disease. In light of this growing health problem, a burgeoning body of research documents the importance of support within the couple relationship for patient achievement of dietary and exercise adherence. Most of this research has focused on the impact of illness-specific support partners provide on patients' diabetes outcomes (e.g., Beverly, Miller, \& Wray, 2008; Stephens, Rook, Franks, Khan, \& Iida, 2010), but research has yet to uncover linkages between general relationship functioning and dietary and exercise adherence. For example, higher marital quality was associated with better dietary and exercise self-care in cross-sectional, but not longitudinal analyses (Trief, Ploutz-Snyder, Britton, \& Weinstock, 2004). The current study proposes that common dyadic coping may be a general relationship process associated with better dietary and exercise adherence indirectly via the mechanism of patient and spouse perceptions of diabetes efficacy. This model is tested using data from 117 married couples in which one person has been diagnosed with type 2 diabetes (patients).

\section{Background}

Dyadic coping is conceptualized as the systemic, interactional process that couples use to manage stress (Bodenmann, 2000; 2005). Common dyadic coping refers to the collective efforts 
of both partners to mitigate the impact of stress and encompasses problem-focused strategies (e.g., searching for solutions together, engaging in serious discussions to think through the problem, and helping each other see problems in a new light) and emotion-focused strategies (e.g., helping each other relax and showing affection to each other). The purpose of common dyadic coping is to resolve a problem together or help each partner reduce emotional arousal.

Our focus on this specific form of coping stems from empirical and theoretical evidence that illness-specific coping efforts are most effective at producing positive outcomes when both partners actively collaborate in illness management (Berg \& Upchurch, 2007; Hagedoorn et al., 2000; Kuijer et al., 2000). Indeed, research exploring illness-specific support in couples coping with diabetes has found a sense of "being in this together," regular communication about the diabetes care regimen, providing encouragement, and joint participation in self-care behaviors are associated with improved patient dietary and exercise adherence (Beverly \& Wray, 2010; Khan, Stephens, Franks, Rook, \& Salem, in press; Stephens et al., in press). We hypothesize that a general pattern of frequently handling stress by working through problems together and helping each other relax would likely influence patient management of type 2 diabetes, which is conceptualized as one specific stressor couples face (e.g., Schokker et al., 2010). Yet, studies examining the link between dyadic coping and individual well-being, including physical health symptoms, have not found substantive direct associations between these constructs (e.g., Bodenmann, Meuwly, \& Kayser, 2011) leading to the conclusion that dyadic coping is more closely related to relationship functioning and individual coping is responsible for health outcomes (Bodenmann, 2000). An alternate possibility is that dyadic coping is indirectly associated with well-being and health promoting behaviors, but what mechanism might link these variables? 
Drawing from social-cognitive theory (Bandura, 1986; 1997), diabetes self-efficacy is proposed as a potential mediator linking common dyadic coping with dietary and exercise adherence. Self-efficacy is defined as “beliefs in one's capabilities to organize and execute courses of action" (Bandura, 1997, p. 3). Diabetes self-efficacy assesses confidence in the ability of the patient to follow the various aspects of the diabetes self-care regimen. In this way, diabetes self-efficacy is a very precise set of cognitions specific to this illness, such as one's level of confidence to exercise regularly, follow the recommended diet, or resist food temptations. Social cognitive theory posits that self-efficacy is a key determinant of behavior and is shaped by a host of factors, including the social environment. For the current study, we propose that frequent use of common dyadic coping to handle stress as a general relationship dynamic (a characteristic indicative of a supportive social environment) would increase patient and partner confidence in the patient's ability to successfully manage a specific stressful health condition, type 2 diabetes, ultimately resulting in increased adherence to a healthful diet and exercise regimen. Existing research provides support for such a model.

Results from the illness-specific coping literature have demonstrated linkages between spouse support and patient diabetes self-efficacy. Qualitative research examining ways spouses of patients with type 2 diabetes support dietary and exercise adherence revealed that when couples worked together on a daily basis to accomplish these goals, patient confidence to eat healthfully and exercise increased (Beverly et al., 2008; Beverly \& Wray, 2010). Other research has found moderated effects between spouse coping efforts and patient self-efficacy. A daily diary study found spousal support increased exercise-specific efficacy when high levels of support are accompanied by high levels of control, such as prompting the patient to engage in exercise and monitoring the patient to ensure that he or she exercised (Khan et al., in press). A 
study of patients diagnosed with type 2 diabetes or asthma found that partners' not openly communicating worries about the patient's illness was actually associated with higher patient self-efficacy when patient symptoms were low (de Ridder, Shcreurs, \& Kuijer, 2005) and overprotective coping efforts from a spouse were associated with less self-efficacy, especially when glycemic control was poor (Schokker et al., 2011). These studies demonstrate strong evidence that patient diabetes self-efficacy is informed by spouses' illness-specific coping behavior (such as encouraging the patient to exercise or eat healthfully), but no research has examined the association between diabetes self-efficacy and the couple's general pattern of coping with stress that is not illness-specific, operationalized as common dyadic coping in the present investigation.

There is extensive evidence linking diabetes self-efficacy with adherence to the diabetic lifestyle regimen in cross-sectional (King et al., 2010) and longitudinal (Nakahara et al., 2006) research, with some studies finding self-efficacy to be the strongest predictor of adherence behaviors when analyzed in concert with other potential salient cognitions (Plotnikoff, Trinh, Courneya, Karunamuni, \& Sigal, 2011; Zulman, Rosland, Choi, Langa, \& Heisler, 2012). In perhaps the most comprehensive examination of the impact of diabetes self-efficacy on adherence behaviors, Nouwen and colleagues (2011) surveyed newly diagnosed patients with type 2 diabetes five times over the course of 18 months to examine the predictive power of constructs derived from social-cognitive theory and self-determination theory on following the recommended diabetic diet. The results indicated dietary self-efficacy was predictive of increased dietary adherence and changes in self-efficacy were predictive of changes in dietary adherence over the 18-month time span, accounting for all related variables in the model.

While there is strong evidence that patient diabetes self-efficacy is related to increased 
dietary and exercise adherence, we could not locate any studies that assessed spouse confidence in the patient's ability to follow the recommended diabetes self-care behaviors. The current study utilizes assessment of patient and spouse report of diabetes efficacy to determine the unique predictive power of each partner's perspective on dietary and exercise adherence, which aligns with calls for increased attention to the beliefs of healthy spouses for understanding illness adjustment of patients (Berg \& Upchurch, 2007). Spousal confidence in patient adherence to the diabetes care regimen could have unique diagnostic power to understand patient dietary and exercise adherence because spouses do not necessarily have access to the same illness-specific information as the patient (e.g., how the patient is feeling) unless it is communicated from the patient. As such, spousal diabetes efficacy might be informed through different, more distal sources of information, such as the couple's general dyadic process for handling stress and could, thus, yield unique insight into illness management.

\section{The Present Study}

Informed by empirical and theoretical literatures related to dyadic coping (e.g., Bodenmann, 2005) and social-cognitive theory (Bandura, 1986; 1997), the current study seeks to test a model linking a specific aspect of intimate relationship functioning (common dyadic coping) to dietary and exercise adherence in patients diagnosed with type 2 diabetes indirectly via the mechanism of patient and spouse diabetes efficacy. To increase confidence in the findings of our model, several control variables will be included in the analyses and three alternative models will be tested. In terms of control variables, comorbid medical conditions, amount of time the patient has been diagnosed with type 2 diabetes, age, gender, and patient and spouse reports of relationship satisfaction will be included. The presence of more comorbid medical conditions in both patient and partner may be linked to less confidence in one's ability to 
follow the intensive lifestyle regimen necessary to manage type 2 diabetes. A leading theoretical framework for couples coping with chronic illness contends that coping behavior, illness beliefs, and illness adjustment change depending on the stage of the illness and the couple's position in the life course (Berg \& Upchurch, 2007), suggesting the need to account for length of diagnosis with type 2 diabetes and patient age in all analyses. Additionally, a study of patients with type 2 diabetes found older age to be associated with higher levels of diabetes self-efficacy and eating less healthy food (Hessler, Fisher, Mullan, Glasgow, \& Masharani, 2011). There are well documented gender disparities in general support provision among couples (e.g., Neff \& Karney, 2005), with female partners typically providing support more precisely when it is needed, and prior research found female partners' well-being across four health domains to be more strongly related to dyadic coping than male partners' well-being (Bodenmann et al., 2011). Finally, prior studies found higher relationship satisfaction to be closely related to better dyadic coping (Bodenmann, 2005) and marital quality was associated with better dietary and exercise self-care in prior cross-sectional analyses (Trief et al., 2004).

The model in this study will also be empirically tested against three plausible alternatives. Diabetes efficacy is conceptualized to fully mediate the associations between common dyadic coping and dietary and exercise adherence, but partial mediation is also a possibility. Specifically, common dyadic coping could exhibit a direct association with patient dietary and exercise self-care. Studies exploring illness-specific coping behavior have documented a direct effect from spousal coping with dietary and exercise adherence (e.g., Khan et al., in press), but the existing evidence examining dyadic coping and health variables have not found direct associations (Bodenmann 2000; 2005). Next, social-cognitive theory specifies bidirectional influences between self-efficacy and social influences, whereas confidence to engage in certain 
behaviors can shape the social environment just as the social environment can shape self-efficacy (Bandura, 1986; 1997). In the current study, higher diabetes efficacy could, therefore, encourage couples to handle general relationship stress in a collective manner, which might then spur greater dietary and exercise adherence. In the same vein, bidirectional influences are also possible between behavior and self-efficacy, which could ultimately influence the social environment. A final alternative model will be considered with dietary and exercise adherence predicting diabetes efficacy, which ultimately predicts common dyadic coping.

This research makes several important contributions to the existing literature exploring the influence of the couple relationship on diabetes outcomes. First, this study explores how general relationship functioning may be associated with patient adherence to the diabetes selfcare regimen. Prior studies have focused on the associations of illness-specific couple interactions with diabetes outcomes, but have yet to uncover how general relationship functioning is connected to exercise or dietary adherence (e.g., Trief et al., 2004). Next, patient and partner report of diabetes efficacy are assessed in this study. There is extensive evidence linking patient self-efficacy to dietary and exercise adherence, but no studies have included partners' perspective on their confidence that the patient will carry out these behaviors. Patients and partners diabetes efficacy could contribute unique information in understanding actual selfcare behavior and would point to the value in partners' cognitions for understanding diabetes outcomes. Finally, common dyadic coping is a specific couple relationship process that, if salient for diabetes outcomes, would provide a precise target in the intimate relationship for intervention efforts aimed at improving adherence to the diabetes lifestyle regimen.

\section{Method}

\section{Procedures}


Participants were recruited from a patient registry at a large Midwest medical center. Several thousand patients were part of the registry, so search criteria selected only patients diagnosed with type 2 diabetes mellitus between the ages of 18-74 who had not experienced an amputation, blindness or low vision, chronic kidney disease or renal failure. A total of 525 patients were selected through this screening process. Next, a research assistant called each patient and first screened them for study eligibility (i.e., diagnosed with type 2 diabetes, was between the ages of 18-74, were currently married, their spouse was not diagnosed with type 2 diabetes, and both the patient and spouse were willing to participate). As a result of this screening process, 180 patients did not qualify and 105 patients could not be contacted after multiple attempts to reach them, leaving 240 eligible patients. Of these 240 eligible patients, 85 declined to participate and 155 consented to participate. After consenting to participate, the research assistant obtained a separate e-mail address for the patient and the patient's spouse and sent each person a unique link to complete an online survey. Study data were collected and managed using REDCap electronic data capture tools hosted at University of Kansas Medical Center (Harris, Taylor, Thielke, Payne, Gonzalez, \& Conde, 2009). If preferred, paper copies of the survey were sent to the participants and returned with a self-addressed stamped envelope that was provided for them. Of the 155 patients that originally consented, 117 patients and spouses completed the survey ( $49 \%$ response rate from the 240 eligible participants). Upon both the patient and spouse completing the survey, the couple was sent $\$ 30$ as a token of appreciation for their participation.

\section{Participants}

Patients had been diagnosed with type 2 diabetes for 10.98 years $(S D=9.19)$, on average. Slightly more diabetic patients were male $(57.3 \%)$ than female $(42.7 \%)$. The majority of patients 
and spouses were European American (87.0\% and 83.3\%, respectively), $7.0 \%$ of patients and $5.3 \%$ of spouses were African American, $3.5 \%$ of patients and $4.4 \%$ of spouses were Latino/a, and the remaining $2.6 \%$ of patients and $7 \%$ of spouses were another race not listed. The average age of patients was 57.44 years $(S D=9.83)$ and was 57.38 years $(S D=10.15)$ for spouses. These couples tended to be in long-term marriages, with the average relationship length reported as 29.40 years $(S D=13.88)$. Nearly two-thirds of participants were in their first marriage $(61.5 \%)$, $27.4 \%$ had been married twice, and $11.1 \%$ had been married three or more times. In terms of patients' education, . $9 \%$ had less than a high school diploma, $12.1 \%$ were high school graduates, 43.1\% completed some college or had an associate's degree/technical training, $21.6 \%$ held a bachelor's degree, and $22.4 \%$ had a postgraduate degree. For spouses' education, $19.1 \%$ were high school graduates (none had less than a high school diploma), 31.3\% completed some college or had an associate's degree/technical training, 28.7\% held a bachelor's degree, and $20.9 \%$ had a postgraduate degree. Only $18.5 \%$ of couples indicated their household income was below $\$ 50,000$ a year, $24.1 \%$ made between $\$ 50,000$ and $\$ 69,999$ annually, $31.5 \%$ earned between $\$ 70,000$ and $\$ 99,999$ per year, and $25.9 \%$ had a household income of $\$ 100,000$ or more per year.

\section{Measures}

Common Dyadic Coping. The 5-item common dyadic coping subscale of the dyadic coping inventory (DCI; Bodenmann, 2008) was used to assess how the couple collectively copes with general life stress. Patients and spouses indicated how often they, as a couple, engaged in a series of activities to deal with stress. Sample items are, "We try to cope with the problem together and search for solutions," and "We help one another to put the problem in perspective and see it in a new light." Responses range from $1=$ very rarely to $5=$ very often and mean 
scores were calculated. Cronbach's alpha reliability for patients was $\alpha=.83$ and $\alpha=.89$ for spouses. The psychometric properties of the DCI have been demonstrated in three language groups, with the underlying factor structure replicated through confirmatory factor analysis and evidence of construct validity appearing through the comparison of the DCI with measures of marital quality (from $r=.51$ to $r=.67$ for common dyadic coping with marital quality) and couple communication (from $r=-.21$ to $r=-.41$ for common dyadic coping with demandwithdraw communication; Ledermann et al., 2010). Correlations between common dyadic coping and relationship satisfaction in the current study are similar in magnitude $(r=.66$ for patients and $r=.71$ for spouses).

Diabetes Efficacy. The 7-item self-efficacy subscale of the Multidimensional Diabetes Questionnaire (MDQ; Talbot, Nouwen, Gingras, Gosselin, \& Audet, 1997) was used to measure diabetes efficacy. This scale assesses the patient's confidence in his or her ability to adhere to various aspects of the diabetic treatment regimen. For spouses, wording of the items was changed to reflect the partner's confidence in the patient's ability to follow the diabetic lifestyle. Sample items include "How confident are you in your ability to follow your diet?" and "How confident are you in your ability to keep your blood sugar under control?" Responses range from $0=$ not at all confident to $5=$ completely confident and mean scores were computed. Cronbach's alpha reliability in the current study for patients was $\alpha=.87$ and $\alpha=.91$ for spouses. Talbot and colleagues provided psychometric information on the MDQ with a sample of adult type 2 diabetes patients. The underlying factor structure of the measure was established with confirmatory factor analysis and construct validity was evident by examining the correlations of the subscales with theoretically relevant covariates. Diabetes self-efficacy was negatively associated with depressive symptoms $(r=-.36)$ and positively with dietary adherence $(r=.58)$, 
exercise adherence $(r=.48)$, and better glycemic control $(r=.28$; Talbot et al., 1997).

Dietary and Exercise Adherence. The 2-item general diet and 2-item exercise subscales of the revised Summary of Diabetes Self-Care Activities (SDSCA) measure (Toobert, Hampson, \& Glasgow, 2000) were used to assess patients' adherence to the dietary and exercise regimens necessary for managing type 2 diabetes. Prior research demonstrated that patient self-report of better diabetes self-management behavior (including exercise and dietary adherence) significantly predicted lower levels of $\mathrm{HbA}_{1 \mathrm{c}}$, a biological indicator of glycemic control, obtained from patient medical records after accounting for a variety of control variables $(\beta=$ $.13, p<.001$; Heisler, Smith, Hayward, Krein, \& Kerr, 2003). Items related to dietary adherence are, "How many of the last seven days have you followed a healthful eating plan?" and "On average, over the past month, how many days per week have you followed your eating plan?" The inter-correlation of these items was $r=.83(p<.001)$. The exercise adherence items are, "On how many of the last seven days did you participate in at least 30 minutes of physical activity?" and "On how many of the last seven days did you participate in a specific exercise session (such as swimming, walking, biking) other than what you do around the house or as part of your work?" The inter-correlation of these items was $r=.70(p<.001)$. Responses range from $0=0$ days to $7=7$ days and mean scores were calculated for each scale. The psychometric properties of the SDSCA have been extensively evaluated (for a review of seven studies, see Toobert et al., 2000). Construct validity for the dietary and exercise adherence subscales has been established through comparison to a variety of longer alternative diet and exercise measurement instruments, including daily diary measures up to 7 days and attendance records for exercise classes (validity coefficient from $r=-.23$ to $r=-.54$ for dietary adherence and $r=.20$ to $r=.58$ for exercise adherence). 
Control Variables. Patient comorbidities, years diagnosed with type 2 diabetes, age, gender, and relationship satisfaction and spouse comorbidities, age, and relationship satisfaction were explored as potential control variables. Comorbidities for the patient and spouse were calculated from the Charlson comorbidity index (Charlson, Pompei, Ales, \& MacKenzie, 1987). This index assesses the presence of a wide range of potential comorbid health conditions, such as heart disease, asthma, ulcers, stroke, and etcetera. Respondents report whether they have experienced the health condition $(1=y e s)$ or not $(0=n o)$. Items were summed to produce a total score reflecting the total number of comorbid health problems. On average, patients reported 2.09 comorbid health problems $(S D=1.82)$, while spouses reported $1.14(S D=1.43)$. Patients reported their age of diagnosis with type 2 diabetes. This was subtracted from their current age to determine their length of diagnosis in years. The 4-item version of the Couples Satisfaction Index (CSI-4; Funk \& Rogge, 2007) was used to assess relationship satisfaction. The CSI-4 is a measure of relationship satisfaction that was developed through an item-response theory analysis of 180 items commonly used to measure relationship satisfaction. Sample items include, "I have a warm and comfortable relationship with my partner," and "In general, how satisfied are you with your relationship?" Responses are measured on Likert scales that vary with each item, but generally range from $0=$ Not at all to $5=$ Completely and mean scores were computed. Cronbach's alpha reliability in the current study is $\alpha=.94$ for patients and spouses.

\section{Analytic Plan}

The data were first explored with descriptive statistics to ensure the data met the statistical assumptions of structural equation modeling and correlations to explore the bivariate associations among the variables in the model. Missing data were low for the variables in this study, ranging from $1.7 \%$ for dietary adherence to $5.1 \%$ for patient report of diabetes self- 
efficacy, and was handled through the full-information maximum likelihood procedure. To provide evidence for the missing at random (MAR) assumption of FIML, correlations were computed between dummy coded study variables reflecting whether the data were missing or present and all other study variables. No correlations were significant, providing evidence that the pattern of missingness is not systematically related to other variables in this study, thus, supporting the MAR assumption. The research questions were answered using structural equation modeling with maximum likelihood estimation in Mplus 7.0 (Muthen \& Muthen, 2012) and estimates standardized on $y$ and $x$ are presented. Model fit was evaluated with the model chisquare $\left(\chi^{2}\right)$, the comparative fit index (CFI), Tucker-Lewis Index (TLI), the root mean square error approximation (RMSEA), and the standardized root mean square residual (SRMR) with a non-significant chi-square, values greater than .95 for CFI and TLI and smaller than .06 and .08 for RMSEA and SRMR suggesting good fit (Hu \& Bentler, 1999). Common dyadic coping is a construct that conceptually and operationally occurs at the dyad level, so patient and partner reports of this variable were modeled as indicators of a latent, couple-level variable following procedures for the common fate model (Ledermann \& Macho, 2009). The alternative models were tested using nested model comparison through the chi-square difference test and non-nested model comparison with the Akaike Information Criterion (AIC) and Bayesian Information Criterion (BIC), where smaller values of the AIC and BIC indicate less discrepancy between the hypothesized model and the true model (West, Taylor, \& Wu, 2012). Finally, the indirect paths were tested with bootstrapping procedures (Preacher \& Hayes, 2008).

\section{Results}

\section{Correlations}

The results of the correlation analysis revealed important information about the bivariate 
relations among the central variables of interest in this study and can be viewed in Table 1. First, patient and spouse reports of common dyadic coping were related to each other's' report $(r=.59$, $p<.001)$, their own diabetes efficacy $(r=.22, p=.021$ for patient and $r=.40, p<.001$ for spouse), and dietary and exercise adherence (from $r=.20, p=.042$ to .21, $p=.029$ ), with the exception of spouse common dyadic coping with dietary adherence $(r=.16, p=.101)$. Patient and spouse diabetes efficacy were related to each other's report $(r=.63, p<.001)$ and patient dietary and exercise adherence (from $r=.45$ to .63 , all $p \mathrm{~s}<.001$ ). Dietary adherence was related to exercise adherence $(r=.41, p<.001)$. With the results of the correlations proceeding as anticipated, the structural equation model analysis can proceed.

\section{Structural Equation Model Results}

The final structural equation model results can be viewed in Figure 1. Initially, all the variables in the model were regressed on each control variable. Non-significant paths from the control variables were trimmed, one at a time to ensure model fit was not significantly reduced, until only the substantive paths and the most parsimonious model remained. Spouse comorbidities and patient gender were not associated with any of the variables in the model and thus, were not included in the final analysis. The final structural equation model fit the data well: $\chi^{2}(28)=41.158, p=.052 ;$ RMSEA $=.063($ C.I. $=.000, .102) ;$ CFI $=.970 ;$ TLI $=.943 ;$ SRMR $=$ .048 , and accounted for $42 \%$ of the variance in dietary adherence and $27 \%$ of the variance in exercise adherence.

Higher levels of common dyadic coping were associated with higher diabetes efficacy for both patients $(\beta=.19, p=.041)$ and spouses $(\beta=.43, p<.001)$. The residual variance of patient and spouse diabetes efficacy was significantly correlated $(\beta=.60, p<.001)$. Patient diabetes self-efficacy was related to better dietary adherence $(\beta=.49, p<.001)$ and demonstrated a trend 
toward significance with exercise adherence $(\beta=.20, p=.062)$. Spouse diabetes efficacy was associated with better dietary adherence $(\beta=.25, p=.012)$ and exercise adherence $(\beta=.33, p=$ .002). In terms of the control variables, patient and partner report of relationship satisfaction were each associated with higher common dyadic coping $(\beta=.45, p<.001$ for patients and $\beta=$ $.52, p<.001$ for spouses). A longer time since diagnosis of type 2 diabetes was associated with lower patient diabetes self-efficacy $(\beta=-.16, p=.029)$ and less exercise adherence $(\beta=-.19, p=$ .015). Patient older age was associated with higher patient diabetes self-efficacy $(\beta=.31, p<$ $.001)$ and having more comorbid medical conditions for patients was associated with less diabetes efficacy in spouses $(\beta=-.38, p<.001)$.

To test whether any associations in the model were significantly stronger for patient or spouse variables, corresponding parameter estimates were constrained to be equal and the chisquare difference test was conducted. The association of common dyadic coping with diabetes efficacy was significantly stronger for spouses $\left(\chi_{\text {diff }}^{2}(1)=9.708, p=.002\right)$, but neither of the spousal associations between diabetes self-efficacy and dietary adherence $\left(\chi_{\text {diff }}^{2}(1)=2.465, p=\right.$ $.116)$ or exercise adherence $\left(\chi_{\text {diff }}^{2}(1)=.263, p=.608\right)$ significantly differed.

\section{Model Comparison}

Next, we compared our proposed model to three theoretically plausible alternatives. First, a nested model comparison was conducted to evaluate whether the indirect link between common dyadic coping and dietary and exercise adherence was best represented as fully mediated by diabetes efficacy (the proposed model) or partially mediated (direct paths from common dyadic coping to the adherence variables). Each model was estimated and the chisquare difference test indicated omitting the direct paths from common dyadic coping to dietary and exercise adherence did not result in a poorer fit to the data $\left(\chi_{\text {diff }}^{2}(2)=1.253, p=.534\right)$, 
supporting the more parsimonious, fully mediated model. Next, our proposed model was tested against a model in which diabetes efficacy was modeled to predict common dyadic coping, which ultimately predicted dietary and exercise adherence and a model testing the reverse causal sequence. The AIC and BIC values were smaller for the proposed model $(\mathrm{AIC}=4702.644$; $\mathrm{BIC}$ $=4683.096)$ than either alternative model: diabetes efficacy $\rightarrow$ common dyadic coping $\rightarrow$ exercise and dietary adherence $(\mathrm{AIC}=4765.960 ; \mathrm{BIC}=4747.210)$ and exercise and dietary adherence $\rightarrow$ diabetes efficacy $\rightarrow$ common dyadic coping $(\mathrm{AIC}=4734.393 ; \mathrm{BIC}=4714.845)$, signifying less discrepancy between our hypothesized model and the true model.

\section{Test of Indirect Effects}

The indirect effect from common dyadic coping to dietary and exercise adherence was tested with 2,000 bootstraps and a 95\% confidence interval. Both indirect paths from common dyadic coping to dietary adherence were significant at the trend level: common dyadic coping $\rightarrow$ patient diabetes self-efficacy $\rightarrow$ dietary adherence $(\beta=.10, p=.071$, C.I. $=.008, .20)$ and common dyadic coping $\rightarrow$ spouse diabetes efficacy $\rightarrow$ dietary adherence $(\beta=.11, p=.057$, C.I. $=.002, .22)$. The effect for patients can be interpreted as follows: a 1 standard deviation unit increase in common dyadic coping is associated with a .10 standard deviation unit increase in dietary adherence via the prior effect on patient diabetes self-efficacy, holding the control variables, partner diabetes efficacy, and exercise adherence constant. One indirect path to exercise adherence was also significant: common dyadic coping $\rightarrow$ spouse diabetes efficacy $\rightarrow$ exercise adherence $(\beta=.14, p=.012$, C.I. $=.03, .25)$.

\section{Discussion}

Drawing from empirical and theoretical literatures related to dyadic coping (Bodenmann, 2000; 2005) and social-cognitive theory (Bandura, 1986; 1997), the purpose of this study was to 
test a model exploring the potential link between common dyadic coping in married couples where one partner is diagnosed with type 2 diabetes and dietary and exercise adherence indirectly through patient and spouse diabetes efficacy.

The first important finding from this study is that common dyadic coping, or the collective efforts of both partners to handle stress, is related to better dietary and exercise adherence via the mechanism of diabetes efficacy. Broadly speaking, this study is the first to document that general relationship functioning is associated with specific dietary and exercise self-care behaviors patients perform to manage type 2 diabetes. Failure to find similar associations in prior research likely stem from the exploration of marital quality as opposed to specific relationship processes (such as dyadic coping) and the investigation of only direct effects (e.g., Trief et al., 2004), which were also not significant in the alternative model tested with the current data. Additionally, prior research that explored the direct influence of dyadic coping on health and well-being outcomes failed to find significant associations (Bodenmann, 2000; Bodenmann et al., 2011). The current study provides an important extension to the literatures exploring couples coping with chronic illness and general dyadic coping by demonstrating the necessity of considering the indirect influence of general dyadic coping on health outcomes in addition to illness-specific coping efforts. Indeed, when couples frequently handled stress through joint discussion and relaxation, patients and partners were more confident in the patient's ability to follow the recommended diabetes self-care regimen, which was ultimately associated with eating more healthfully and participating in more frequent physical activity. Future research should continue to elucidate the indirect mechanisms by which the couple relationship is associated with type 2 diabetes outcomes. Prior studies have found marital quality to be associated with less diabetes distress (Trief, Wade, Britton, \& Weinstock, 2002) and 
better diabetes quality of life (Trief, Himes, Orendorff, \& Weinstock, 2001). These variables, among others, may also prove to be mechanisms linking the couple relationship with diabetes self-care outcomes.

This study is also the first to explore partner perceptions of diabetes efficacy in addition to patient report of diabetes self-efficacy. As anticipated from prior research with patients (e.g., Nouwen et al., 2011; Zulman et al., 2012), both patient and partner diabetes efficacy were related to better dietary and exercise adherence (albeit, at the trend level for patient self-efficacy to exercise adherence). The application of equality constraints indicated that patient and spouse self-efficacy were equally related to actual patient dietary and exercise adherence, which is quite remarkable. These findings highlight the importance of assessing partners' confidence in the patient's ability to follow through on the type 2 diabetes self-care regimen for understanding actual dietary and exercise adherence. Additionally, the bootstrap test of the indirect effects revealed that patient and spouse diabetes efficacy differentially linked common dyadic coping to dietary and exercise adherence, with patient and spouse efficacy serving as the mechanism for healthful eating (at the trend level) and only spouse efficacy connecting exercise adherence. As research continues exploring the connections between the couple relationship and diabetes outcomes, spouses' diabetes cognitions merit exploration in addition to patients' beliefs.

The results from common dyadic coping to patient and spouse diabetes efficacy are also interesting, as the link between these variables was significantly stronger for spouses than patients. While a diagnosis of type 2 diabetes for one partner in a marital relationship is certainly a stressor for both members of the dyad, its impact is likely more directly felt by the patient. As such, patient confidence in following all aspects of the self-care regimen is likely informed by a wider variety of factors outside the couple relationship as compared to their spouse. Prior 
research has shown diabetes distress, feeling burdened following the prescribed diabetes selfcare behaviors, and poorer glycemic control $\left(\mathrm{HbA}_{1 \mathrm{c}}\right)$ are all associated with less patient diabetes self-efficacy (Nakahara et al., 2006). As this is the first study to explore spouses' diabetes efficacy, further exploration is needed to determine other factors that determine spouse diabetes efficacy, but this study highlights the importance of including intimate relationship functioning for understanding spouse confidence in the patient's ability to follow the diabetes diet and exercise regimen.

These results also have important theoretical implications. First, the non-nested model comparisons supported the proposed sequence of associations: common dyadic coping $\rightarrow$ diabetes efficacy $\rightarrow$ dietary and exercise adherence. Social-cognitive theory specifies bidirectional influences between behavior, self-efficacy and social influences, however. The superiority of the proposed model could be related to how these variables are operationalized. The social influence of interest in this study refer to behaviors couples use to handle stress in general and self-efficacy pertains to confidence in one dyad members ability to handle a specific stressor, the type 2 diabetes regimen. It is plausible that the effect of such a precise self-efficacy operationalization would be small on a general social influence, such as common dyadic coping, and may not have been detected with this relatively small sample size. If the social influence under examination was also specific to the illness, such as partners' illness-specific coping efforts (e.g., Berg \& Upchurch, 2007; Hagedoorn et al., 2000; Kuijer et al., 2000; Stephens et al., 2010), the bidirectional association with diabetes efficacy might be more pronounced. In terms of dyadic coping, the indirect association between common dyadic coping and individual management of a specific stressor suggests the possibility of a cascade effect (e.g., Cox, MillsKoonce, Propper, \& Gariepy, 2010), whereas the effective management of stress between the 
dyad (i.e., effective dyadic coping) might trigger a ripple of cognitive (i.e., diabetes efficacy) and behavioral mechanisms that might ultimately lead to more successful management of specific stressors faced by the individuals in the relationship, (i.e., better dietary and exercise adherence). This exciting possibility needs to be examined longitudinally and warrants investigation across a variety of stressors and health conditions.

Lastly, results from this study can also be used to inform intervention efforts aimed at improving patient adherence to the diabetes lifestyle regimen by demonstrating that common dyadic coping is a specific couple relationship process that, if improved, might indirectly improve dietary and exercise adherence. There is an existing couple intervention protocol that specifically targets dyadic coping, Couples Coping Enhancement Training (CCET; Bodenmann \& Shantinath, 2004). Outcome studies of CCET demonstrate marked gains in marital quality evident after one year follow-up (Bodenmann, Charvoz, Cina, \& Widmer, 2001) and improved dyadic coping that persist two years later (Bodenmann, Perrez, Cina, \& Widmer, 2002). Research shedding light on potential mechanisms of change in the couple relationship that might impact diabetes outcomes is important, as the only randomized control trial to date that has tested a couple intervention for weight-loss in patients with type 2 diabetes was largely ineffective (Wing, Marcus, Epstein, \& Jawad, 1991). This intervention focused only on dietary adherence and losing weight, not couple relationship functioning. Perhaps, future couple interventions to improve diabetes outcomes would prove more helpful if elements from CCET were incorporated in the treatment.

\section{Limitations}

There are some limitations of this study that must be taken into account when interpreting these findings. The main limitation of this study stems from the cross-sectional nature of the data 
utilized. Although the proposed model is grounded in strong theoretical and empirical literature, the temporal ordering among these variables cannot be established with these data, even though two non-nested model comparisons of an alternative causal sequence indicated the superiority of our proposed model. The model in this study, along with plausible alternatives, must be tested in future studies with longitudinal dyadic data. Second, the constructs of interest were measured with self-report instruments. Thus, a portion of the observed associations between these variables could stem from shared method variance. Measurement of common dyadic coping, in particular, could also be assessed with observational techniques in future research, as the current study assesses perceptions of this behavioral construct. The use of latent variable modeling to capture only the shared variance between patient and spouse reports, however, provides more confidence that the dyadic construct is analytically represented. Third, the primary variables of interest in this study were common dyadic coping, diabetes efficacy, and dietary and exercise adherence, but there are other variables salient to relationship functioning, diabetes cognitions, and diabetes outcomes that may serve to link general functioning in the intimate relationship to successful type 2 diabetes adjustment. This research demonstrates the importance of exploring indirect connections between the couple relationship and type 2 diabetes outcomes and future research could further the understanding of these connections through the exploration of other variables in each domain. Finally, this study is comprised of a relatively small sample of predominately White married couples recruited from one diabetes clinic in the Midwestern United States, potentially limiting the generalizability of these findings. Additional research is needed with more diverse samples of couples in other types of relationships, including dating, cohabiting, and same-sex relationships.

\section{Conclusion}


This study demonstrates the importance of couples' collective efforts to handle life stress for understanding the dietary and exercise components of type 2 diabetes self-care through the mechanism of patient and spouse diabetes efficacy. While these findings must be replicated with more diverse samples and longitudinal design, this research provides the first evidence that general relationship functioning is associated with successful adherence to healthful eating and frequency of exercise. Additionally, this study demonstrates the importance of assessing diabetes efficacy in spouses, not just patient perceptions, as spouses' diabetes efficacy was the only mechanism serving to link common dyadic coping with exercise adherence. It seems that patient and spouse diabetes cognitions, therefore, provide unique insight into different self-care behaviors. Intervention and assessment efforts aiming to improve diabetes outcomes may be enhanced by targeting patients' dynamics in their intimate relationship and eliciting spouses' beliefs about type 2 diabetes, in addition to patients'. 


\section{References}

Berg, C. A., \& Upchurch, R. (2007). A developmental-contextual model of couples coping with chronic illness across the adult life span. Psychological Bulletin, 133, 920-954. doi: 10.1037/0033-2909.133.6.920

Beverly, E. A., Miller, C. K., \& Wray, L. A. (2008). Spousal support and food-related behavior change in middle-aged and older adults living with type 2 diabetes. Health Education \& Behavior, 35, 707-720. doi: 10.1177/1090198107299787

Beverly, E. A., \& Wray, L. A. (2010). The role of collective efficacy in exercise adherence: A qualitative study of spousal support and type 2 diabetes management. Health Education Research, 25, 211-223. doi: 10.1093/her/cyn032

Bandura, A. (1997). Self-efficacy: The exercise of control. New York: W. H. Freeman and Company.

Bandura, A. (1986). Social foundations of thought and action: A social cognitive theory. Englewood Cliffs, NJ: Prentice Hall.

Bodenmann, G. (2000). Stress and coping in couples. Göttingen, Germany: Hogrefe.

Bodenmann, G. (2005). Dyadic coping and its significance for marital functioning. In T.

Revenson, K. Kayser, \& G. Bodenmann (Eds.), Couples coping with stress: Emerging perspectives on dyadic coping (pp. 35-50). Washington, DC: American Psychological Association.

Bodenmann, G. (2008). Dyadic Coping Inventory (DCI): Test Manual. Bern, Göttingen: Huber \& Hogrefe Testverlag.

Bodenmann, G. Charvoz, L., Cina, A., \& Widma, K. (2001). Prevention of marital distress by enhancing the coping skills of couples: 1-year follow-up study. Swiss Journal of 
Psychology, 60, 3-10. doi: 10.1024//1421-0185.60.1.3

Bodenmann, G., Meuwly, N., \& Kayser, K. (2011). Two conceptualizations of dyadic coping and their potential for predicting relationship quality and individual well-being: A comparison. European Psychologist, 16, 255-266. doi: 10.1027/1016-9040/a000068

Bodenmann, G., Perrex, M., Cina, A., Widmer, K. (2002). The effectiveness of a coping-focused prevention approach: A two-year longitudinal study. Swiss Journal of Psychology, 61, 195-202. doi: 10.1024/1421-0185.61.4.195

Bodenmann, G., \& Shantinath, S. D. (2004). The couples coping enhancement training (CCET): A new approach to prevention of marital distress based upon stress and coping. Family Relations, 53, 477-484. doi: 10.1111/j.0197-6664.2004.00056.x

Centers for Disease Control and Prevention. (2011). National diabetes fact sheet: National estimates and general information on diabetes and prediabetes in the United States, 2011. Atlanta, GA: U.S. Department of Health and Human Services.

Charlson, M. E., Pompei, P., Ales, K. L., \& MacKenzie, C. R. (1987). A new method of classifying prognostic comorbidity in longitudinal studies: Development and validation. Journal of Chronic Diseases, 40, 373-383.

Cox, M. J., Mills-Koonce, R., Propper, C., \& Gariepy, J. L. (2010). Systems theory and cascades in developmental psychopathology. Development and Psychopathology, 22, 497-506. doi: $10.1017 /$ S09545794100000234

Danaie, G., Finucane, M. M., Lu, Y., Singh, G. M., Cowan, M. J., \& Paciorek, C. J., et al. (2011). National, regional, and global trends in fasting plasma glucose and diabetes prevalence since 1980: Systematic analysis of health examination surveys and epidemiological studies with 370 country-years and 2.7 million participants. The Lancet, 
378, 1-10. doi: 10.1016/S0140-6736(11)60679-X

de Ridder, D. T. D., Schreurs, K. M. G., \& Kuijer, R. G. (2005). Is spousal support always helpful to patients with asthma or diabetes? A prospective study. Psychology and Health, 20, 497-508. doi: 10.1080/147683205000098699

Funk, J. L., \& Rogge, R. D. (2007). Testing the ruler with item response theory: Increasing precision of measurement for relationship satisfaction with the couples satisfaction index. Journal of Family Psychology, 21, 572-583. doi: 10.1037/0893-3200.21.4.572

Hagedoorn, M., Kuijer, R. G., Buunk, B. P., De Jong, G. M., Wobbes, Th., \& Sanderman, R. (2000). Marital satisfaction in patients with cancer: Does support from intimate partners benefit those who need it the most? Health Psychology, 19, 274-282. doi: 10.1037//02786133.19 .3 .274

Harris, P. A., Taylor, R., Thielke, R., Payne, J., Gonzalez, N., \& Conde, J. G. (2009). Research electronic data capture (REDCap): A metadata-driven methodology and workflow process for providing translational research informatics support. Journal of Biomedical Informatics, 42, 377-381. doi:10.1016/j.jbi.2008.08.010

Heisler, M., Smith, D. M., Hayward, R. A., Krein, S. L., \& Kerr, E. A. (2003). How well do patients' assessments of their diabetes self-management correlate with actual glycemic control and receipt of recommended diabetes services? Diabetes Care, 26, 738-743. doi: 10.2337/diacare.26.3.738

Hessler, D. M., Fisher, L., Mullan, J. T., Glasgow, R. E., \& Masharani, U. (2011). Patient age: A neglected factor when considering disease management in adults with type 2 diabetes. Patient Education and Counseling, 2011, 154-159. doi: 10.1016/j.pec.2010.10.030

Hu, L., \& Bentler, P. M. (1999). Cutoff criteria for fit indexes in covariance structure analysis: 
Conventional criteria versus new alternatives. Structural Equation Modeling, 6, 1-55. doi: $10.1080 / 10705519909540118$

Khan, C. M., Stephens, M. A. P., Franks, M. M., \& Rook, K. S. (in press). Influences of spousal support and control on diabetes management through physical activity. Health Psychology. doi: 10.1037/a0028609

King, D. K., Glasgow, R. E., Toobert, D. J., Strycker, L. A., Estabrooks, P. A., Osuna, D., \& Faber, A. J. (2010). Self-efficacy, problem solving, and social-environmental support are associated with diabetes self-management behaviors. Diabetes Care, 33, 751-753. doi: $10.2337 / \mathrm{dc} 09-1746$

Kuijer, R. G., Ybema, J. F., Buunk, B. P., de Jong, G. M., Thijs-Boer, F., \& Sanderman, R. (2000). Active engagement, protective buffering, and overprotection: Three ways of giving support by intimate partners of patients with cancer. Journal of Social and Clinical Psychology, 19, 256-275. doi: 10.1521/jscp.2000.19.2.256

Ledermann, T., Bodenmann, G., Gagliardi, S., Charvoz, L., Verardi, S., Rossier, J., Bertoni, A., \& Iafrate, R. (2010). Psychometrics of the dyadic coping inventory in three language groups. Swiss Journal of Psychology, 69, 201-212. doi: 10.1024/1421-0185/a000024.

Ledermann, T., \& Macho, S. (2009). Mediation in dyadic data at the level of the dyads: A structural equation modeling approach. Journal of Family Psychology, 23, 661-670. doi: 10.1037/a0016197.

Muthén, L.K. and Muthén, B.O. (1998-2012). Mplus User's Guide. Seventh Edition. Los Angeles, CA: Muthén \& Muthén.

Nakahara, R., Yoshiuchi, K., Kumano, H., Hara, Y., Suematsu, H., \& Kuboki, T. (2006). Prospective study on influence of psychosocial factors on glycemic control in Japanese 
patients with type 2 diabetes. Psychosomatics, 47, 240-246. doi:

10.1176/appi.psy.47.3.240

Neff, L. A., \& Karney, B. R. (2005). Gender differences in social support: A question of skill or responsiveness? Journal of Personality and Social Psychology, 88, 79-90. doi: $10.1037 / 0022-3514.88 .1 .79$

Nouwen, A., Ford, T., Balan, A. T., Twisk, J., Ruggiero, L., \& White, D. (2011). Longitudinal motivational predictors of dietary self-care and diabetes control in adults with newly diagnosed type 2 diabetes mellitus. Health Psychology, 30, 771-779. doi:

$10.1037 / \mathrm{a} 0024500$

Plotnikoff, R. C., Trinh, L., Courneya, K. S., Karunamuni, N., \& Sigal, R. J. (2011). Predictors of physical activity in adults with type 2 diabetes. American Journal of Health Behavior, 35, 359-370.

Preacher, K . J., \& Hayes, A. F. (2008). Asymptotic and resampling strategies for assessing and comparing indirect effects in multiple mediator models. Behavior Research Methods, 40, 879-891. doi: 10.3758/BRM.40.3.879

Schokker, M. C., Links, T. P., Bouma, J., Keers, J. C., Sanderman, R., Wolffenbuttel, B. H. R., Hagedoorn, M. (2011). The role of overprotection by the partner in coping with diabetes: A moderated mediation model. Psychology and Health, 26, 95-111. doi: $10.1080 / 08870440903342325$

Schokker, M. C., Stuive, I., Bouma, J., Keers, J.C., Links, T. P., Wolffenbuttel, B. H. R., Sanderman, R., \& Hagedoorn, M. (2010). Support behavior and relationship satisfaction in couples dealing with diabetes: main and moderating effects. Journal of Family Psychology, 24, 578-586. doi: 10.1037/a0021009 
Stephens, M. A. P., Franks, M. M., Rook, K. S., Iida, M., Hemphill, R., \& Salem, J. K. (in press). Spouses' attempts to regulate day-to-day dietary adherence among patients with type 2 diabetes. Health Psychology. doi: 10.1037/a0030018

Stephens, M. A. P., Rook, K. S., Franks, M. M., Khan, C. M. Iida, M. (2010). Spouses' use of social control to improve diabetic patients' dietary adherence. Families, Systems, \& Health, 28, 199-208. doi: 10.1037/a0020513

Talbot, F., Nouwen, A., Gingras, J., Gosselin, M., \& Audet, J. (1997). The assessment of diabetes-related cognitive and social factors: The multidimensional diabetes questionnaire. Journal of Behavioral Medicine, 20, 291-312. doi: 10.1023/A:1025508928696

Toobert, D. J., Hampson, S. E., \& Glasgow, R. E. (2000). The summary of diabetes self-care activities measure: Results from 7 studies and a revised scale. Diabetes Care, 23, 943950. doi: $10.2337 /$ diacare.23.7.943

Trief, P. M., Himes, C. L., Orendorff, R., \& Weinstock, R. S. (2001). The marital relationship and psychosocial adaption and glycemic control of individuals with diabetes. Diabetes Care, 24, 1384-1389. doi: 10.2337/diacare.24.8.1384

Trief, P. M., Ploutz-Snyder, R., Britton, K. D., \& Weinstock, R. S. (2004). The relationship between marital quality and adherence to the diabetes care regimen. Annals of Behavioral Medicine, 27, 148-154. doi: 10.1207/s15324796abm2703_2

Trief, P. M., Wade, M. J., Britton, K. D., \& Weinstock, R. S. (2002). A prospective analysis of marital relationship factors and quality of life in diabetes. Diabetes Care, 25, 1154-1158. doi: $10.2337 /$ diacare.25.7.1154

West, S. G., Taylor, A. B., \& Wu, W. (2012). Model fit and model selection in structural 
equation modeling. In R. Hoyle (Ed.), Handbook of structural equation modeling (pp. 209-231). New York, NY: The Guilford Press.

Wing, R. R., Goldstein, M. G., Acton, K. J., Birch, L. L., Jakicic, J. M., \& Sallis, J. F., et al. (2001). Behavioral science research in diabetes: Lifestyle changes related to obesity, eating behavior, and physical activity. Diabetes Care, 24, 117-123. doi:

10.2337/diacare.24.1.117

Wing, R. R., Marcus, M. D., Epstein, L. H., \& Jawad, A. (1991). A “family-based” approach to the treatment of obese type II diabetic patients. Journal of Consulting and Clinical Psychology, 59, 156-162. doi: 10.1037/0022-006X.59.1.156

Zulman, D. M., Rosland, A. M., Choi, H., Langa, K. M., \& Heisler. M. (2012). The influence of diabetes psychosocial attributes and self-management practices on changes in in diabetes status. Patient Education and Counseling, 87, 74-80. doi: 10.1016/j.pec.2011.07.013 
Table 1

Correlations and Descriptive Statistics for Study Variables ( $n=117$ couples)

\begin{tabular}{lccccccc}
\hline Variable & 1 & 2 & 3 & 4 & $M$ & $S D$ & Range \\
\hline 1. Dyadic Coping & $.59^{* * *}$ & $.22^{*}$ & $.20^{*}$ & $.20^{*}$ & 3.20 & .84 & $1-5$ \\
2. Diabetes Efficacy & $.40^{* * *}$ & $.63^{* * *}$ & $.63^{* * *}$ & $.43^{* * *}$ & 2.52 & 1.10 & $0-5$ \\
3. Dietary Adherence & .16 & $.54^{* * *}$ & - & $.41^{* * *}$ & 4.50 & 1.90 & $0-7$ \\
4. Exercise Adherence & $.21^{*}$ & $.45^{* * *}$ & $.41^{* * *}$ & - & 2.84 & 2.30 & $0-7$ \\
$M$ & 3.24 & 2.71 & & & & & \\
$S D$ & .94 & 1.20 & & & & &
\end{tabular}

Note: Patient scores are above the diagonal, spouses are below the diagonal, and between patient and spouses are along the diagonal.

${ }^{*} p<.05 . * * * p<.001$ (two-tailed). 
Figure 1

Final Model of the Indirect Association Between Common Dyadic Coping and Dietary and Exercise Adherence via Patient and Spouse Diabetes Efficacy ( $n=117$ couples)

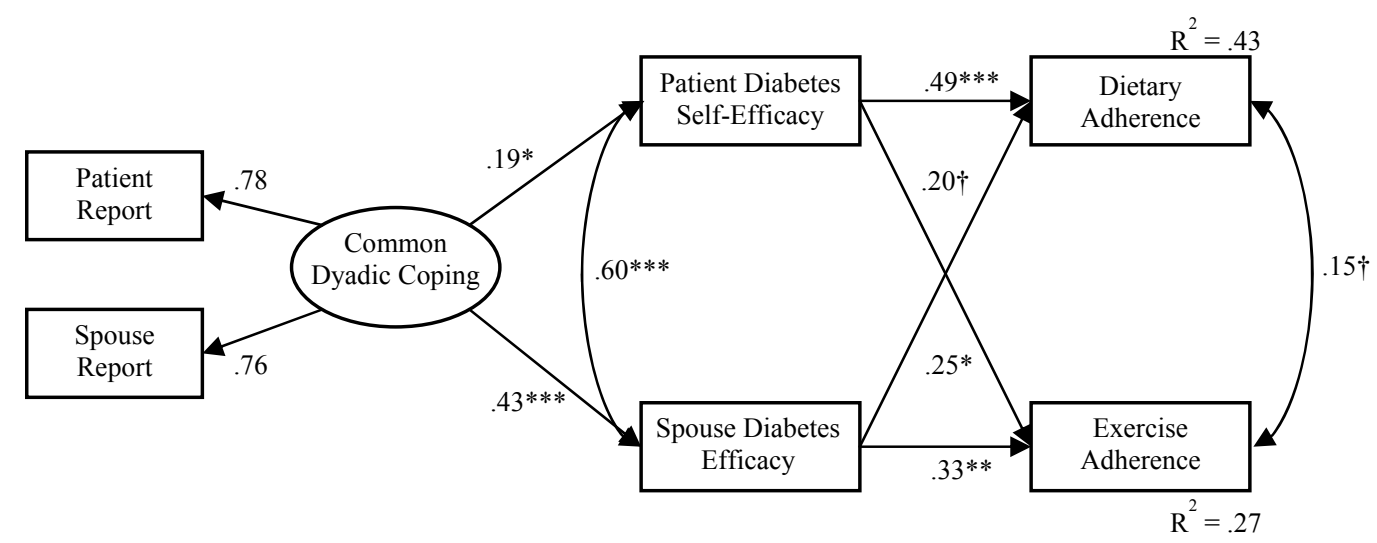

Note: Standardized estimates shown. Patient comorbidities, total years diagnosed with Type 2 diabetes, and age and patient and partner relationship satisfaction were included as control variables, but are not included in the figure for clarity. Model fit indices: $\chi^{2}(28)=41.158, p=$ $.052 ; \mathrm{RMSEA}=.063($ C.I. $=.000, .102) ; \mathrm{CFI}=.970 ; \mathrm{TLI}=.943 ; \mathrm{SRMR}=.048$

$\dagger p<.10 . * p<.05 . * * p<.01 . * * * p<.001$ (two-tailed). 